\title{
基于表型性状构建中国花生地方品种骨干种质
}

\author{
间彩霞 ${ }^{1}$ 王 娟 ${ }^{1} \quad$ 张 $^{\text {浩 }^{1}}$ 李春娟 $^{1} \quad$ 宋秀霞 $^{2} \quad$ 孙全喜 $^{1}$ 苑翠玲 $^{1}$ \\ 赵小波 ${ }^{1}$ 单世华 ${ }^{1, *}$
}

${ }^{1}$ 山东省花生研究所, 山东青岛 $266100 ;{ }^{2}$ 菏泽市牡丹区农业农村局, 山东菏泽 274000

摘 要: 中国花生地方品种遗传多样性丰富, 是花生新品种选育的重要亲本来源。本研究以种质库保存的 2741 份地 方种质为材料, 基于种植区划和植物学类型分组, 平方根法确定取样量, 组内按 13 个表型数据进行 UPGMA 聚类分 析, 类内随机取样, 构建骨干种质。利用 $t$ 检验、 $F$ 测验、卡方测验、极差、表型保留比例、表型相关性等对骨干种 质代表性进行检验和评价; 并利用主成分分析和直方图对骨干种质进行确认。结果表明, 构建了包含 259 份种质的中 国花生地方品种骨干种质, 占全部种质的 9.4\%, 包括多粒型 14 份、珍珠豆型 85 份、龙生型 42 份、普通型 103 份、 中间型 15 份。在 $P<0.05$ 概率条件下, 骨干种质 13 个性状的均值、方差、变异系数、香农指数与全部种质无显著 差异, 且保留了全部种质的分布范围、表型保留比例和表型相关性; 二者的植物学类型组成和生态分布是一致的, 具 有相似的遗传结构和分布频率。建立的骨干种质很好地代表了全部种质的遗传变异和群体结构, 可为花生种质创新 和优异等位基因发掘奠定良好的基础。

关键词：花生；地方品种；表型性状；骨干种质；代表性评价

\section{Developing the key germplasm of Chinese peanut landraces based on pheno- typic traits}

\author{
YAN Cai-Xia ${ }^{1}$, WANG Juan ${ }^{1}$, ZHANG Hao ${ }^{1}$, LI Chun-Juan ${ }^{1}$, SONG Xiu-Xia ${ }^{2}$, SUN Quan-Xi ${ }^{1}$, YUAN \\ Cui-Ling ${ }^{1}$, ZHAO Xiao-Bo ${ }^{1}$, and SHAN Shi-Hua ${ }^{1, *}$ \\ ${ }^{1}$ Shandong Peanut Research Institute, Qingdao 266100, Shandong, China; ${ }^{2}$ Agricultural and Rural Bureau of Mudan District, Heze 274000, Shan- \\ dong, China
}

\begin{abstract}
Chinese peanut landraces are important parent resources in peanut breeding due to their abundant genetic diversity. In this study, a total of 2741 original accessions from peanut seed bank were divided into 26 groups based on their botanical variety and ecological distribution. The key accessions were established based on the analysis of 13 phenotypic traits by the square root strategy, UPGMA clustering within groups and random sampling in individual clusters, and evaluated by $t$-test, $F$-test, Chi-squared test, ranging, the ratio of phenotypic retention, and phenotypic correlation analyses. Finally, the principal components analysis (PCA) and the histogram analysis were used to re-confirm the key germplasm. The total of 259 as a key germplasm was selected, accounting for $9.4 \%$ of total accessions, which included 14 of var. fastigiata, 85 of var. vulgaris, 42 of var. hirsuta, 103 of var. hypogaea and 15 of irregular type. There were no significant differences $(P<0.05)$ in means, variance, coefficient of variation, and Shannon-weaver diversity index for 13 phenotypic traits between key germplasm and entire collection. The key germ-
\end{abstract}

本研究由泰山学者特聘专家项目(ts201712080), 中央引导地方科技发展专项资金, 农业科研杰出人才及其创新团队培养(13190194), 青岛市民生科技计划项目(17-3-3-49-nsh)，山东省农业良种工程项目(2017LZN033，2017LZGC003)，山东省农业产业技术体系项目 (SDAIT-04-02)和山东省农业科学院农业科技创新工程项目(CXGC2016A01)资助。

This study was supported by the Taishan Scholars Project (ts201712080), the Central Guidance for Local Science and Technology, the Outstanding Talents and Innovation Team in Agricultural Research (13190194), the Qingdao Science and Technology Plan for the Public Benefit (17-3-3-49-nsh), the Fine Breeding Project of Shandong Province (2017LZN033, 2017LZGC003), the Shandong Agriculture Research System (SDAIT-04-02), and the Agricultural Science and Technological Innovation Project of Shandong Academy of Agricultural Science (CXGC2016A01).

*通信作者(Corresponding author): 单世华, E-mail: shansh1971@163.com, Tel: 0532-87629307

第一作者联系方式: E-mail: cxyan335@sina.com, Tel: 0532-87626756

Received (收稿日期): 2019-07-17; Accepted (接受日期): 2019-12-26; Published online (网络出版日期): 2020-01-17.

URL: http://kns.cnki.net/kcms/detail/11.1809.S.20200117.1338.004.html 
plasm preserved the distribution range, the ratio of phenotypic retention and the phenotypic correlation of primary collection, with similar composition of botanical variety and ecological distribution. PCA and the histogram confirmed the homogeneity of genetic structure and distribution frequency between two collections. Thus, this key germplasm can represent the genetic variability and population structure of entire collection, and enhance innovation of peanut genetic resources and exploitation of elite alleles.

Keywords: peanut; landrace; phenotypic traits; key germplasm; representative evaluation

花生栽培种(Arachis hypogaea L.)起源于南美洲, 隶属于豆科花生属(Arachis), 一年生, 异源四倍体 $(\mathrm{AABB})$, 推测是由二倍体野生种经过自然杂交和染 色体加倍而形成的 ${ }^{[1]}$ 。自多途径引进后, 在我国多变 的气候及复杂的地理环境下形成了遗传多样性较为 丰富的种质资源。目前国家中期种质库中的保有量

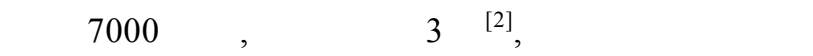
4638份, 因其变异广泛、适应性好、配合力高及对 病害、虫害及逆境等的抗性, 是花生品种改良的重 要亲本来源。追溯建国以来育成花生品种的系谱, 有 25 个地方品种直接或间接参与育成了 $85 \%$ 的品种, 其中 $40 \%$ 的亲缘来源于伏花生、徐州 68-4、狮头企、 粤油551、徐州402和粤选58。地方品种成为花生育 种的“骨干亲本”[3], 但是, 在育种实践中用作亲本的 不足百份, 特别是多粒型种质和龙生型种质有效利 用甚少 ${ }^{[4]}$ 。同时, 花生育种存在着严重的“近亲繁殖”, 导致品种遗传基础狭窄，适应性、抗病(逆)性减退， 产量上很难取得重要突破, 因此, 拓宽现有花生品 种的遗传基础已迫在眉眏。

地方品种具有丰富的多样性, 这为品种选育和 遗传研究提供了广阔的遗传基础。然而, 其庞大的 资源数量反而阻碍了作物的品种改良和新品种选 育。构建核心种质(core collection)或骨干种质(key germplasm), 即以最小的资源份数最大限度地代表 整个资源的遗传多样性 ${ }^{[5]}$, 无疑是解决这一矛盾的 有效策略, 这为地方种质的深入评价、创新利用和 基因挖掘开辟了新的途径。迄今为止, 基于表型性 状和分子标记数据, 已构建了水稻、小麦、玉米、 大豆、棉花、燕麦、谷子、芝麻、糜子、㯟稷等 ${ }^{[6]}$
多种作物的核心(骨干)种质。核心种质的取样比例、 取样策略以及有效性评价等理论研究也取得了进展, 为核心(骨干)种质的构建及代表性评价提供了坚实 的理论依据。

作物的表型多样性是遗传多样性的外在表现, 国 内外研究机构均利用表型数据构建了花生核心种质, 如美国的831份核心种质 ${ }^{[7]}$ 和 112 份微核心种质 ${ }^{[8]}$, 国 际半干旱地区热带作物研究所(ICRISAT)的1704份核 心种质 ${ }^{[9]}$ 和 184 份微核心种质 ${ }^{[10]}$, 姜慧芳等 ${ }^{[2]}$ 构建的 298份小核心种质, 并且开展了大量的性状评价与抗 病(虫或逆)性鉴定 ${ }^{[11-17]}$ 。本研究以建国以来收集保存的 2741 份代表性地方品种为材料, 以13个表型性状数据 为基础, 构建中国花生地方品种骨干种质, 对其代表 性进行评价, 为地方品种的保护与利用、花生种质的 创新利用及品种的遗传改良提供理论依据。

\section{1 材料与方法}

\section{1 材料}

2741 份有明确地理来源的中国栽培花生地方品 种, 其有关数据来自山东省花生研究所编写的 《中 国花生品种资源目录》及中国农业科学院油料作物 研究所编写的 《中国花生品种资源目录(续编一、 二)》。涉及生育期、百果重、百仁重、出仁率、株 高、株型、开花习性、分枝型、植物学类型 9 个农 艺性状和粗蛋白含量、粗脂肪含量、油酸含量、亚 油酸含量 4 个品质性状。质量性状根据其表型进行 赋值(表 1), 数量性状数据采用 0.5 个标准差为间距 进行标准化, 分为 10 级 ( 1 级 $\leq X-2 \delta, 10$ 级 $>X+2 \delta$, 中间每级间差 $0.5 \delta, X$ 为性状平均值, $\delta$ 为标准差)。

\section{表 1 花生质量性状赋值}

Table1 Quantified value of four qualitative traits in peanut

\begin{tabular}{ll}
\hline \multicolumn{1}{c}{ 性状 Trait } & \multicolumn{1}{c}{ 赋值 Quantified value } \\
\hline 株型 & 直立 $=1$, 半蔓 $=3$, 匍旬 $=5$ \\
Plant type & Erect $=1$, Semi-spreading $=3$, Spreading $=5$ \\
开花习性 & 交替 $=1$, 连续 $=3$ \\
Flowering habit & Alternate flowering $=1$, Continuous flowering $=3$ \\
分枝型 & 密枝 $=1$, 疏枝 $=3$ \\
Branching pattern & Dense branch $=1$, Sparse branch $=3$ \\
植物学类型 & 多粒型 $=1$, 珍珠豆型 $=2$, 龙生型 $=3$, 普通型 $=4$, 中间型 $=5$ \\
Botanical variety & var. fastigiata $=1$, var. vulgaris $=2$, var. hirsute $=3$, var. hypogaea $=4$, Irregular type $=5$ \\
\hline
\end{tabular}




\section{2 分组及取样比例的确定}

根据中国栽培花生的七大地理种植区划(黄河 流域花生区、长江流域花生区、东南沿海花生区、 云贵高原花生区、黄土高原花生区、东北花生区和 西北花生区)和五大植物学类型(多粒型、珍珠豆型、 龙生型、普通型、中间型)分组。采取常用的平方根 法(square root strategy, S 法)系统取样, 即分组取样 量由整个组内资源份数的平方根值占各组平方根之 和的比例来决定。

$$
N_{i}=N \times \sqrt{n_{i}} / \sum_{j=1}^{m} \sqrt{n_{j}}
$$

其中, $N_{i}$ 为第 $i$ 组的取样数, $n_{i}$ 为第 $i$ 组的品种数, $n_{j}$ 为第 $j$ 组的品种数, $m$ 为总分组数, $N$ 为总品种数。

\section{3 聚类分析}

采用 SPSS 22.0 统计分析软件, 以组为单位采 用类平均数法(UPGMA)进行各性状的系统聚类分 析, 类内利用 Microsoft Excel 提供的 Randbetween 函数随机取样。

\section{4 骨干种质的代表性检测}

利用 SPSS 22.0 中的 $t$ 测验、 $F$ 测验分别判断全 部种质和骨干种质 13 个性状的均值、方差、变异系 数及 Shannon-Weaver 多样性指数 $\left(H^{\prime}\right)$ 是否有差异, 卡平方 $\left(\chi^{2}\right)$ 测验用于检验两者的植物学类型组成、生 态分布及 13 个性状的表型分布频率是否一致。变异 系数 $(\mathrm{CV})=($ 标准偏差 $\mathrm{SD} /$ 平均值 Mean $) \times 100 \%$, 用 于比较全部种质和骨干种质的离散程度。

$$
H^{\prime}=-\sum_{i=1}^{n} P_{i} \ln P_{i}
$$

其中, $P_{i}$ 表示某性状第 $i$ 级的分布频率, $n$ 为总分级数, 用于评价 2 个群体的遗传多样性水平。极差分析和 表型相关性分析分别用于确定全部种质的变异范围 和性状相关性是否在骨干种质中得到了相应保持。 表型保留比例(the ratio of phenotypic retention, RPR) 用于检测骨干种质中是否保留了全部种质足够的 变异。

$$
\mathrm{RPR}=\sum_{i=1}^{n} M_{i} / \sum_{i=1}^{n} M_{i o}
$$

其中, $M_{i o}$ 为全部种质中某性状的表型变异数, $M_{i}$ 为 骨干种质中该性状的表型变异数, $n$ 为性状总数)。

\section{5 骨干种质的确认}

利用主成分分析和直方图比较全部种质与骨干 种质基于主成分的样品分布图及 13 个性状的分布 频率, 对构建的骨干种质有效性进行确认。

\section{2 结果与分析}

2.1 骨干种质的构建、植物学组成及生态分布 全部花生种质被分成了 26 组, 每组种质从 1 到 623 份不等。每组的取样量为 1 47 份。UPGMA 法 聚类分析后随机取样, 得到 242 份种质, 初步评价 后补充极值材料和特殊性状种质 17 份，提取 $9.4 \%$ 的 中国花生地方品种构建骨干种质。该骨干种质包含 全部种质的植物学类型, 其中多粒型 14 份(5.4\%)、 珍珠豆型 85 份 $(32.8 \%$ )、龙生型 42 份(16.2\%)、普通 型 103 份(39.8\%)、中间型 15 份(5.8\%)。卡方测验表 明, 骨干种质代表了全部种质的植物学类型组成 $\left(\chi^{2}=1.600, P=0.809\right)$ (表 2)。另外，骨干种质包含了全 部种质的生态分布, 其中黄河流域花生区 75 份 (29.0\%)、长江流域花生区 75 份(29.0\%)、东南沿海 花生区 63 份(24.3\%)、云贵高原花生区 16 份 $(6.2 \%)$ 、 黄土高原花生区 13 份(5.0\%)、东北花生区 15 份 (5.8\%)和西北花生区 2 份( $0.8 \%)$, 骨干种质代表了 全部种质的生态分布 $\left(\chi^{2}=5.232, P=0.514\right)$, 其中, 西北花生区由于种质较少，仅有的 2 份种质被全部 取样(表 2)。

\section{2 全部种质与骨干种质的平均值、方差和极差} 比较

$t$ 测验表明, 4 个形态性状和 9 个数量性状的平 均值在全部种质与骨干种质中无显著差异, 且骨干 种质中植物学类型、百果重和亚油酸含量这 3 个性 状的平均值都大于全部种质的平均值(表 3)。 $F$ 测验 表明, 除出仁率的方差在全部种质与骨干种质中差 异显著外, 其余 12 个性状的方差均为齐性(表 3)。此 外, 骨干种质大部分性状的方差高于全部种质, 表 明骨干种质遗传几余度明显减小, 变异率更高。极 差分析表明, 全部种质 4 个形态性状的变异范围 100\%保留在骨干种质中; 除百仁重 (保留范围 $64.3 \%$ )、出仁率(保留范围 77.2\%)外, 其余 7 个性状 变异范围的 $86 \%$ 100\%保留在骨干种质中(表 4)。由 此可见, 骨干种质对全部种质的性状的变异幅度具 有良好的代表性。

\section{3 变异系数和 Shannon-Weaver 多样性指数的 比较}

Shannon-Weaver 多样性指数和变异系数常用来 比较不同样品的表型特征、等位基因的丰富度和均 匀度。从表 4 可以看出, 全部种质和骨干种质 13 个 
表 2 骨干种质和全部种质的植物学类型组成、生态分布及其卡方测验

Table 2 Composition of botanical variety, ecological distribution, and Chi-square test in the entire collection and key germplasm

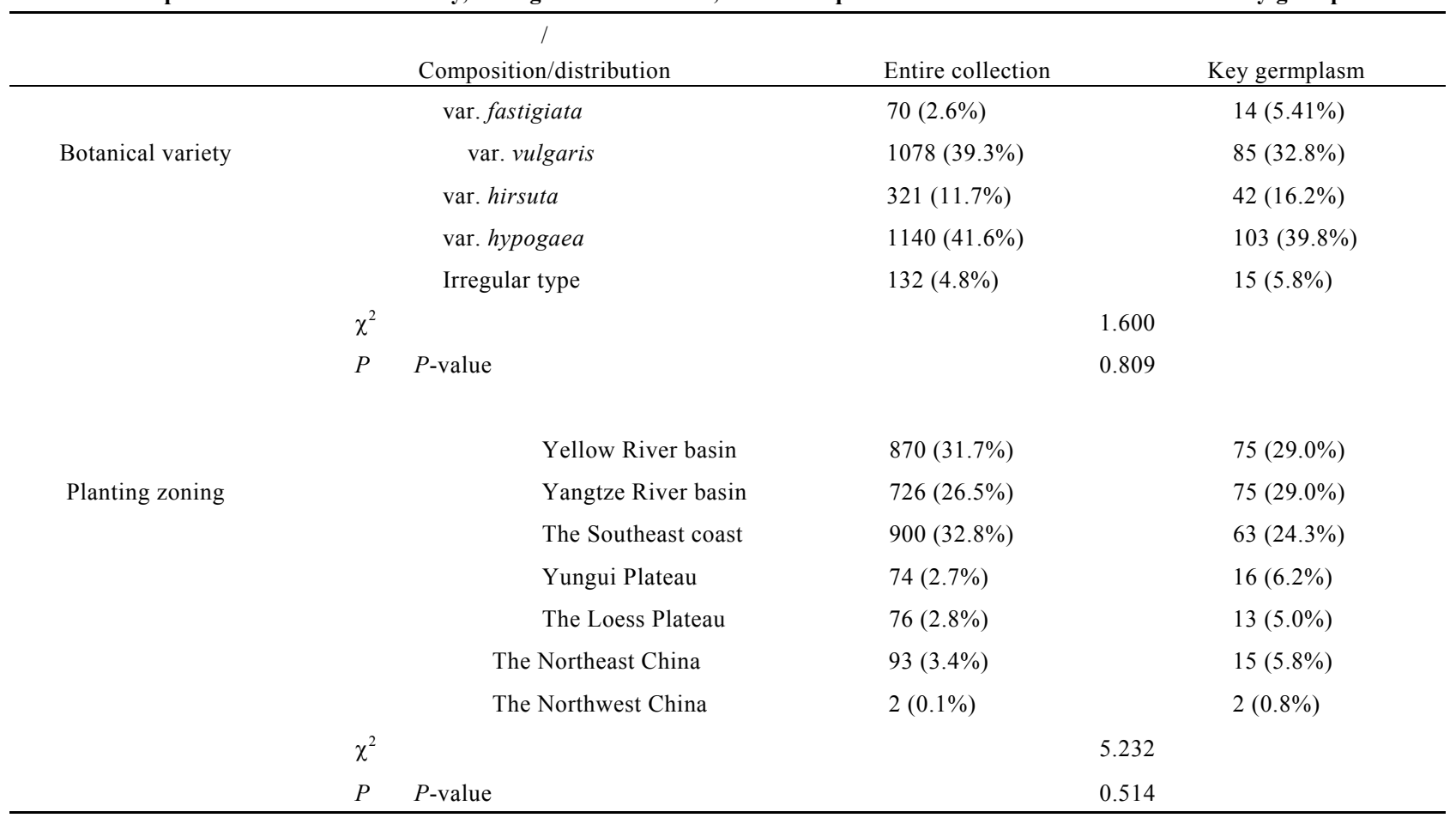

$P>0.05$ 表明差异不显著。 $P>0.05$ means the difference is insignificant.

表 3 全部种质与骨干种质 13 个性状平均值和方差的比较

Table 3 Comparison of means and variances for thirteen traits in the entire collection and key germplasm

\begin{tabular}{|c|c|c|c|c|c|c|c|}
\hline \multirow[b]{2}{*}{$\begin{array}{l}\text { 性状 } \\
\text { Trait }\end{array}$} & \multicolumn{3}{|c|}{ 平均值 Mean } & \multicolumn{4}{|c|}{ 方差 Variance } \\
\hline & $\begin{array}{c}\text { 全部种质 } \\
\text { Entire } \\
\text { collection }\end{array}$ & $\begin{array}{c}\text { 骨干种质 } \\
\text { Key } \\
\text { germplasm }\end{array}$ & $\begin{array}{c}\text { 显著性 } \\
\text { Significance }\end{array}$ & $\begin{array}{c}\text { 全部种质 } \\
\text { Entire } \\
\text { collection }\end{array}$ & $\begin{array}{c}\text { 骨干种质 } \\
\text { Key } \\
\text { germplasm }\end{array}$ & $\begin{array}{c}F \text {-值 } \\
F \text {-value }\end{array}$ & $\begin{array}{c}P \text { 值 } \\
P \text {-value }\end{array}$ \\
\hline 植物学类型 Botanical variety & 3.068 & 3.077 & NS & 1.100 & 1.172 & 0.019 & 0.891 \\
\hline 株型 Plant type & 2.315 & 2.413 & NS & 3.177 & 3.313 & 0.710 & 0.400 \\
\hline 开花习性 Flowering habit & 1.941 & 1.919 & NS & 0.998 & 0.997 & 0.122 & 0.727 \\
\hline 分枝型 Branching pattern & 0.913 & 0.425 & NS & 1.297 & 0.873 & 1.509 & 0.219 \\
\hline 生育期 Growth period & 139.610 & 138.750 & NS & 250.093 & 254.771 & 0.697 & 0.404 \\
\hline 株高 Plant height & 44.074 & 42.376 & NS & 328.256 & 311.667 & 1.293 & 0.256 \\
\hline 百果重 100-pod weight & 158.906 & 159.467 & NS & 2244.396 & 2536.806 & 0.032 & 0.857 \\
\hline 百仁重 100 -seed weight & 64.015 & 63.452 & NS & 372.911 & 415.674 & 0.196 & 0.658 \\
\hline 出仁率 Shelling percentage & 72.477 & 71.890 & NS & 17.460 & 24.461 & 4.352 & 0.037 \\
\hline 粗蛋白含量 Protein content & 27.987 & 27.536 & NS & 11.531 & 15.519 & 3.604 & 0.058 \\
\hline 粗脂肪含量 Oil content & 50.120 & 49.950 & NS & 10.426 & 16.950 & 0.585 & 0.444 \\
\hline 油酸含量 Oleic acid content & 46.400 & 46.370 & NS & 40.424 & 44.819 & 0.047 & 0.829 \\
\hline 亚油酸含量 Linoleic acid content & 33.801 & 33.942 & NS & 30.800 & 32.812 & 0.123 & 0.725 \\
\hline
\end{tabular}

$P>0.05$ 表示差异不显著, $P<0.05$ 表示差异显著, NS 表示差异不显著。

$P>0.05$ means the difference is insignificant; $P<0.05$ means the difference is significant; NS: not significant.

性状的 $H^{\prime}$ 和变异系数是非常相似的。全部种质的平均 $H^{\prime}$ 为 $1.631 \pm 0.153$, 变异系数为 $29.009 \pm 5.964$; 骨干种 质的这 2 个指标分别为 $1.672 \pm 0.154$ 和 $30.125 \pm 5.765$, 骨干种质略高于全部种质。成对双样本 $t$ 测验表明, 骨
干种质 $H^{\prime}$ 极显著高于全部种质 $(P=0.002)$, 变异系数在 2 个群体中差异不显著 $(P=0.123)$, 说明骨干种质的样 本足够大, 且有效去除了总资源中的几余, 保留了全 部种质的遗传多样性, 变异均匀度显著提高。 
表 4 全部种质与骨干种质极差、变异系数和遗传多样性指数的比较

Table 4 Comparison of range, coefficient of variation, and $H^{\prime}$ for thirteen traits in the entire collection and key germplasm

\begin{tabular}{|c|c|c|c|c|c|c|}
\hline \multirow[b]{2}{*}{$\begin{array}{l}\text { 性状 } \\
\text { Trait }\end{array}$} & \multicolumn{2}{|c|}{ 极差 Range } & \multicolumn{2}{|c|}{ 变异系数 CV (\%) } & \multicolumn{2}{|c|}{ 香农多样性指数 $H^{\prime}$} \\
\hline & $\begin{array}{c}\text { 全部种质 } \\
\text { Entire } \\
\text { collection }\end{array}$ & $\begin{array}{c}\text { 骨干种质 } \\
\text { Key } \\
\text { germplasm }\end{array}$ & $\begin{array}{c}\text { 全部种质 } \\
\text { Entire } \\
\text { collection }\end{array}$ & $\begin{array}{c}\text { 骨干种质 } \\
\text { Key } \\
\text { germplasm }\end{array}$ & $\begin{array}{c}\text { 全部种质 } \\
\text { Entire } \\
\text { collection }\end{array}$ & $\begin{array}{c}\text { 骨干种质 } \\
\text { Key } \\
\text { germplasm }\end{array}$ \\
\hline 植物学类型 Botanical variety & $1-5$ & $1-5$ & 34.18 & 35.19 & 1.22 & 1.33 \\
\hline 株型 Plant type & $1-5$ & $1-5$ & 76.97 & 75.43 & 0.87 & 0.90 \\
\hline 开花习性 Flowering habit & $1-3$ & $1-3$ & 51.43 & 52.04 & 0.69 & 0.69 \\
\hline 分枝型 Branching pattern & $1-3$ & $1-3$ & 54.54 & 51.34 & 0.67 & 0.69 \\
\hline 生育期 Growth period & $100-180$ & $100-180$ & 11.33 & 11.50 & 1.88 & 1.94 \\
\hline 株高 Plant height & $7.9-95.0$ & $7.9-93.8$ & 34.18 & 41.66 & 2.07 & 2.05 \\
\hline 百果重 100-pod weight & $45.2-356.0$ & $52.0-324.3$ & 29.81 & 31.58 & 2.02 & 2.05 \\
\hline 百仁重 100 -seed weight & $25.0-190.1$ & $25.0-131.2$ & 30.17 & 32.13 & 1.84 & 1.90 \\
\hline 出仁率 Shelling percentage & $50.37-95.2$ & $51.3-85.9$ & 5.77 & 6.88 & 1.98 & 2.08 \\
\hline 粗蛋白含量 Protein content & $13.7-52.7$ & $13.7-48.7$ & 12.13 & 14.31 & 1.96 & 2.01 \\
\hline 粗脂肪含量 Oil content & $22.1-61.2$ & $24.8-60.6$ & 6.44 & 8.24 & 1.94 & 2.00 \\
\hline 油酸含量 Oleic acid content & $28.5-72.8$ & $29.4-72.8$ & 13.74 & 14.44 & 2.02 & 2.03 \\
\hline 亚油酸含量 Linoleic acid content & $12.6-50.7$ & $12.6-45.6$ & 16.42 & 16.88 & 2.04 & 2.06 \\
\hline 平均值 Average & & & $29.009 \pm 5.964$ & $30.125 \pm 5.765$ & $1.631 \pm 0.153$ & $1.671 \pm 0.154$ \\
\hline$P$ 值 $P$-value & & & 0.1 & & 0.0 & 02 \\
\hline
\end{tabular}

$P>0.05$ 表明差异不显著, $P<0.01$ 表明差异极显著。

$P>0.05$ means the difference is insignificant; $P<0.01$ means the difference is positively significant.

\section{4 分布频率和表型保留比例的比较}

从表 5 可以看出, 全部种质 13 个性状的 102 个 表型分级均包含在骨干种质中。对 2 个群体 13 个性 状的分布频率进行 Chi 平方测验, 差异均不显著, 表明 2 个样本的性状分布是一致的，骨干种质可代
表全部种质的变异。植物学类型、百仁重和粗脂肪 含量的表型保留比例较大, 是补充了一些特殊种质 和极值材料所致; 其余 10 个性状的表型保留比例均 比较合适，表明骨干种质保留了全部种质丰富的变 异, 且丰度更高。

表 5 全部种质与骨干种质 13 个性状的分布频率和表型保留比例的比较

Table 5 Comparison of frequency distribution and the ratio of phenotypic retention for thirteen traits in the entire collection and key germplasm

\begin{tabular}{|c|c|c|c|c|}
\hline $\begin{array}{l}\text { 性状 } \\
\text { Trait }\end{array}$ & $\begin{array}{c}\text { 级数 } \\
\text { Number of classes }\end{array}$ & $\begin{array}{c}\text { Chi-square 值 } \\
\chi^{2} \\
\end{array}$ & $\begin{array}{c}P \text { 值 } \\
P \text {-value } \\
\end{array}$ & $\begin{array}{c}\text { 表型保留比例 } \\
\text { RPR } \\
\end{array}$ \\
\hline 植物学类型 Botanical variety & 5 & 14.078 & 0.007 & 1.30 \\
\hline 株型 Plant type & 3 & 0.723 & 0.697 & 1.01 \\
\hline 开花习性 Flowering habit & 2 & 0.122 & 0.727 & 1.00 \\
\hline 分枝型 Branching pattern & 2 & 1.509 & 0.219 & 1.03 \\
\hline 生育期 Growth period & 10 & 7.851 & 0.549 & 1.15 \\
\hline 株高 Plant height & 10 & 3.264 & 0.917 & 0.98 \\
\hline 百果重 100-pod weight & 10 & 3.289 & 0.952 & 1.13 \\
\hline 百仁重 100 -seed weight & 10 & 11.810 & 0.224 & 2.07 \\
\hline 出仁率 Shelling percentage & 10 & 9.030 & 0.435 & 1.15 \\
\hline 粗蛋白含量 Protein content & 10 & 6.993 & 0.638 & 1.08 \\
\hline 粗脂肪含量 Oil content & 10 & 11.778 & 0.226 & 1.20 \\
\hline 油酸含量 Oleic acid content & 10 & 2.729 & 0.974 & 1.06 \\
\hline 亚油酸含量 Linoleic acid content & 10 & 1.659 & 0.996 & 1.04 \\
\hline
\end{tabular}

$P>0.05$ 表明差异不显著, $P<0.01$ 表明差异极显著。RPR：表型保留比例。

$P>0.05$ means the difference is insignificant; $P<0.01$ means the difference is positively significant. RPR: the ratio of phenotypic retention. 


\section{5 表型相关分析}

一个具有代表性的骨干种质除应具有较小的 遗传冗余外, 还应保留原群体固有的性状间的遗 传关联。对 13 个性状的表型相关性分析表明, 64 对性状在全部种质中呈极显著正相关，其中有 59 对与骨干种质中的相关性是一致的, 39 对在骨干 种质保持了极显著正相关(表 6), 因此, 骨干种质 较好地保持了全部种质的表型相关性。不同性状
间的极显著相关性意味着在育种实践中，可以不 直接篮选难测量的性状，而优先篮选与其极显著 相关的易测量性状，从而较快地实现育种目的。比 如, 由于分枝型和油酸含量极显著负相关(全部种 质中 $r=-0.789^{* *}$ ，骨干种质 $\left.r=-0.685^{* *}\right)$, 那么在 杂交后代中要获得高油酸的单株, 就可以优先篮 选分枝较多的单株, 直到形成稳定的品系后, 再 测定其油酸含量。

表 6 在全部种质和骨干种质中均显著相关的性状

Table 6 Traits significantly correlated in both entire collection and key germplasm

\begin{tabular}{|c|c|c|}
\hline $\begin{array}{l}\text { 性状 } \\
\text { Trait }\end{array}$ & $\begin{array}{c}\text { 全部种质 } \\
\text { Entire collection }\end{array}$ & $\begin{array}{c}\text { 骨干种质 } \\
\text { Key germplasm }\end{array}$ \\
\hline 生育期-百果重 Growth period-100-pod weight & $0.334^{* *}$ & $0.195^{* *}$ \\
\hline 生育期-百仁重 Growth period-100-seed weight & $0.356^{* *}$ & $0.171^{* *}$ \\
\hline 生育期-出仁率 Growth period-shelling percentage & $-0.086^{* *}$ & $-0.171^{* *}$ \\
\hline 生育期-开花习性 Growth period-flowering habit & $-0.736^{* *}$ & $-0.558^{* *}$ \\
\hline 生育期-株型 Growth period-plant type & $0.609^{* *}$ & $0.552^{* *}$ \\
\hline 生育期-株高 Growth period-plant height & $-0.219^{* *}$ & -0.145 \\
\hline 生育期-粗蛋白含量 Growth period-protein content & $-0.267^{* *}$ & $-0.308^{* *}$ \\
\hline 生育期-粗脂肪含量 Growth period-oil content & $-0.185^{* *}$ & -0.030 \\
\hline 生育期-油酸含量 Growth period-oleic acid content & $0.296^{* *}$ & 0.128 \\
\hline 生育期-亚油酸含量 Growth period-linoleic acid content & $-0.279^{* *}$ & -0.077 \\
\hline 生育期-植物学类型 Growth period-botanical variety & $0.630^{* *}$ & $0.441^{* *}$ \\
\hline 生育期-分枝型 Growth period-branching pattern & $-0.851^{* *}$ & $-0.801^{* *}$ \\
\hline 百果重-百仁重 100-pod weight-100-seed weight & $0.908^{* *}$ & $0.873^{* *}$ \\
\hline 百果重-粗脂肪含量 100-pod weight-oil content & $-0.187^{* *}$ & -0.010 \\
\hline 百果重-株高 100-pod weight-plant height & $-0.162^{* *}$ & 0.087 \\
\hline 百果重-油酸含量 100-pod weight-oleic acid content & $0.168^{* *}$ & 0.111 \\
\hline 百果重-亚油酸含量 100-pod weight-linoleic acid content & $-0.209^{* *}$ & $-0.154^{* *}$ \\
\hline 百果重-株型 100-pod weight-plant type & $0.068^{* *}$ & -0.056 \\
\hline 百果重-开花习性 100-pod weight-flowering habit & $-0.359^{* *}$ & $-0.249^{* *}$ \\
\hline 百果重-分枝型 100-pod weight-branching pattern & $-0.479^{* *}$ & $-0.431^{* *}$ \\
\hline 百果重-植物学类型 100 -pod weight-botanical variety & $0.469^{* *}$ & $0.362^{* *}$ \\
\hline 百仁重-粗脂肪含量 100-pod weight-oil content & $-0.193^{* *}$ & -0.026 \\
\hline 百仁重-油酸含量 100-pod weight-oleic acid content & $0.189^{* *}$ & 0.119 \\
\hline 百仁重-亚油酸含量 100-seed weight-linoleic acid content & $-0.225^{* *}$ & $-0.159^{*}$ \\
\hline 百仁重-株高 100-seed weight-plant height & $-0.139^{* *}$ & 0.109 \\
\hline 百仁重-株型 100-seed weight-plant type & $0.071^{* *}$ & -0.092 \\
\hline 百仁重-开花习性 100-seed weight-flowering habit & $-0.404^{* *}$ & $-0.286^{* *}$ \\
\hline 百仁重-分枝型 100-seed weight-branching pattern & $-0.515^{* *}$ & $-0.485^{* *}$ \\
\hline 百仁重-植物学类型 100-seed weight-botanical variety & $0.533^{* *}$ & $0.481^{* *}$ \\
\hline 粗蛋白含量-粗脂肪含量 Protein content-oil content & $-0.423^{* *}$ & $-0.582^{* *}$ \\
\hline 粗蛋白含量-株型 Protein content-plant type & $-0.186^{* *}$ & $-0.185^{* *}$ \\
\hline 粗蛋白含量-株高 Protein content-plant height & $0.091^{* *}$ & 0.007 \\
\hline 粗蛋白含量-开花习性 Protein content-flowering habit & $0.172^{* *}$ & $0.209^{* *}$ \\
\hline 粗蛋白含量-植物学类型 Protein content-botanical variety & $-0.265^{* *}$ & $-0.232^{* *}$ \\
\hline
\end{tabular}


(续表 6)

\begin{tabular}{|c|c|c|}
\hline $\begin{array}{l}\text { 性状 } \\
\text { Trait }\end{array}$ & $\begin{array}{c}\text { 全部种质 } \\
\text { Entire collection }\end{array}$ & $\begin{array}{c}\text { 骨干种质 } \\
\text { Key germplasm }\end{array}$ \\
\hline 粗脂肪含量-油酸含量 Oil content-oleic acid content & $0.064^{* *}$ & 0.080 \\
\hline 粗脂肪含量-亚油酸含量 Oil content-linoleic acid content & $-0.083^{* *}$ & $-0.135^{*}$ \\
\hline 粗脂肪含量-出仁率 Oil content-shelling percentage & $0.141^{* *}$ & 0.048 \\
\hline 粗脂肪含量-株高 Oil content-plant height & $0.110^{* *}$ & 0.142 \\
\hline 粗脂肪含量-开花习性 Oil content-flowering habit & $0.133^{* *}$ & -0.027 \\
\hline 粗脂肪含量-分枝型 Oil content-branching pattern & $0.321^{* *}$ & 0.194 \\
\hline 粗脂肪含量-植物学类型 Oil content-botanical variety & $-0.147^{* *}$ & -0.063 \\
\hline 油酸含量-亚油酸含量 Oleic acid content-linoleic acid content & $-0.932^{* *}$ & $-0.930^{* *}$ \\
\hline 油酸含量-株型 Oleic acid content-plant type & $0.428^{* *}$ & $0.307^{* *}$ \\
\hline 油酸含量-株高 Oleic acid content-plant height & $-0.082^{* *}$ & -0.064 \\
\hline 油酸含量-开花习性 Oleic acid content-flowering habit & $-0.509^{* *}$ & $-0.343^{* *}$ \\
\hline 油酸含量-植物学类型 Oleic acid content-botanical variety & $0.333^{* *}$ & $0.172^{*}$ \\
\hline 油酸含量-分枝型 Oleic acid content-branching pattern & $-0.789^{* *}$ & $-0.685^{* *}$ \\
\hline 亚油酸含量-出仁率 Linoleic acid content-shelling percentage & $-0.107^{* *}$ & -0.121 \\
\hline 亚油酸含量-株型 Linoleic acid content-plant type & $-0.398^{* *}$ & $-0.282^{* *}$ \\
\hline 亚油酸含量-开花习性 Linoleic acid content-flowering habit & $0.486^{* *}$ & $0.314^{* *}$ \\
\hline 亚油酸含量-分枝型 Linoleic acid content-branching pattern & $0.781^{* *}$ & $0.768^{* *}$ \\
\hline 亚油酸含量-植物学类型 Linoleic acid content-botanical variety & $-0.273^{* *}$ & -0.121 \\
\hline 株高-植物学类型 Plant height-botanical variety & $-0.322^{* *}$ & $-0.272^{* *}$ \\
\hline 株高-开花习性 Plant height-flowering habit & $0.357^{* *}$ & $0.287^{* *}$ \\
\hline 株高-株型 Plant height-plant type & $-0.292^{* *}$ & $-0.350^{* *}$ \\
\hline 株型-开花习性 Plant type-flowering habit & $-0.685^{* *}$ & $-0.692^{* *}$ \\
\hline 株型-分枝型 Plant type-branching pattern & $-0.600^{* *}$ & $-0.725^{* *}$ \\
\hline 株型-植物学类型 Plant type-botanical variety & $0.417^{* *}$ & $0.350^{* *}$ \\
\hline 开花习性-分枝型 Flowering habit-branching pattern & $0.966^{* *}$ & $0.965^{* *}$ \\
\hline 开花习性-出仁率 Flowering habit-shelling percentage & $0.076^{* *}$ & 0.014 \\
\hline 植物学类型-开花习性 Botanical variety-flowering habit & $-0.719^{* *}$ & $-0.625^{* *}$ \\
\hline 植物学类型-出仁率 Botanical variety-shelling percentage & $-0.116^{* *}$ & -0.079 \\
\hline 分枝型-出仁率 Branching pattern-shelling percentage & $0.220^{* *}$ & 0.091 \\
\hline 分枝型-植物学类型 Branching pattern-botanical variety & $-0.820^{* *}$ & $-0.660^{* *}$ \\
\hline
\end{tabular}

${ }^{* *}$ 表示相关达到 0.01 极显著性水平, ${ }^{*}$ 表示相关达到 0.05 显著水平。

${ }^{* *}$ indicates significant correlation at $P \leq 0.01 ;{ }^{*}$ indicates significant correlation at $P \leq 0.05$.

\section{6 骨干种质的确认}

利用主成分分析对所构建的骨干种质进行确

认，从骨干种质和全部种质基于第 1 、第 2 主成分的 样品分布图可知(图1), 全部种质中大量的样品集 中在散点图的左右方并存在较重的相互重叠, 表 明这些样品存在较高的遗传相似性, 群体的遗传 几余程度高。9.4\%的平方根法取样后, 骨干种质样 品分布的重叠程度得到了显著降低, 但仍保留了 全部种质的几何形状和特征, 且较多外围的个体 入选到骨干种质中, 表明建立的骨干种质既去除
了全部种质的大部分遗传冗余, 又确保了骨干种 质的代表性。此外, 绘制了 4 个植物学性状与 9 个数 量性状表型分布的直方图, 可以看出, 植物学类 型、开花习性、株型和分枝型 4 个植物学性状在全 部种质和骨干种质中的分布非常吻合; 9个数量性 状在全部种质和骨干种质中都表现出广泛的变异, 且大部分变异的分布频率较为一致, 均基本符合 正态分布(图2)。因此, 骨干种质很好地保留了全 部种质的遗传多样性和群体结构, 确保了骨干种 质的有效性。 

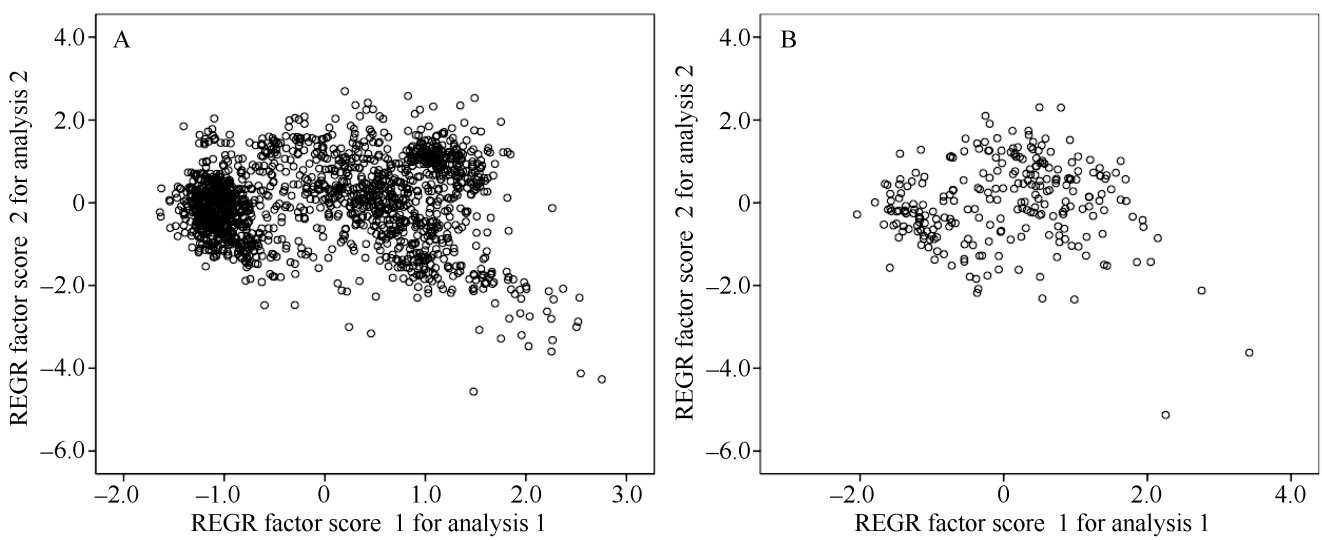

图 1 全部种质与 $9.4 \%$ 取样比例骨干种质的样品主成分分布图

Fig. 1 Principal component plots for the entire collection and key germplasm at $9.4 \%$ sampling proportion

A：全部种质的样品分布图; B: 骨干种质的样品分布图。

A: scatter diagram for entire collection; B: scatter diagram for the key germplasm.
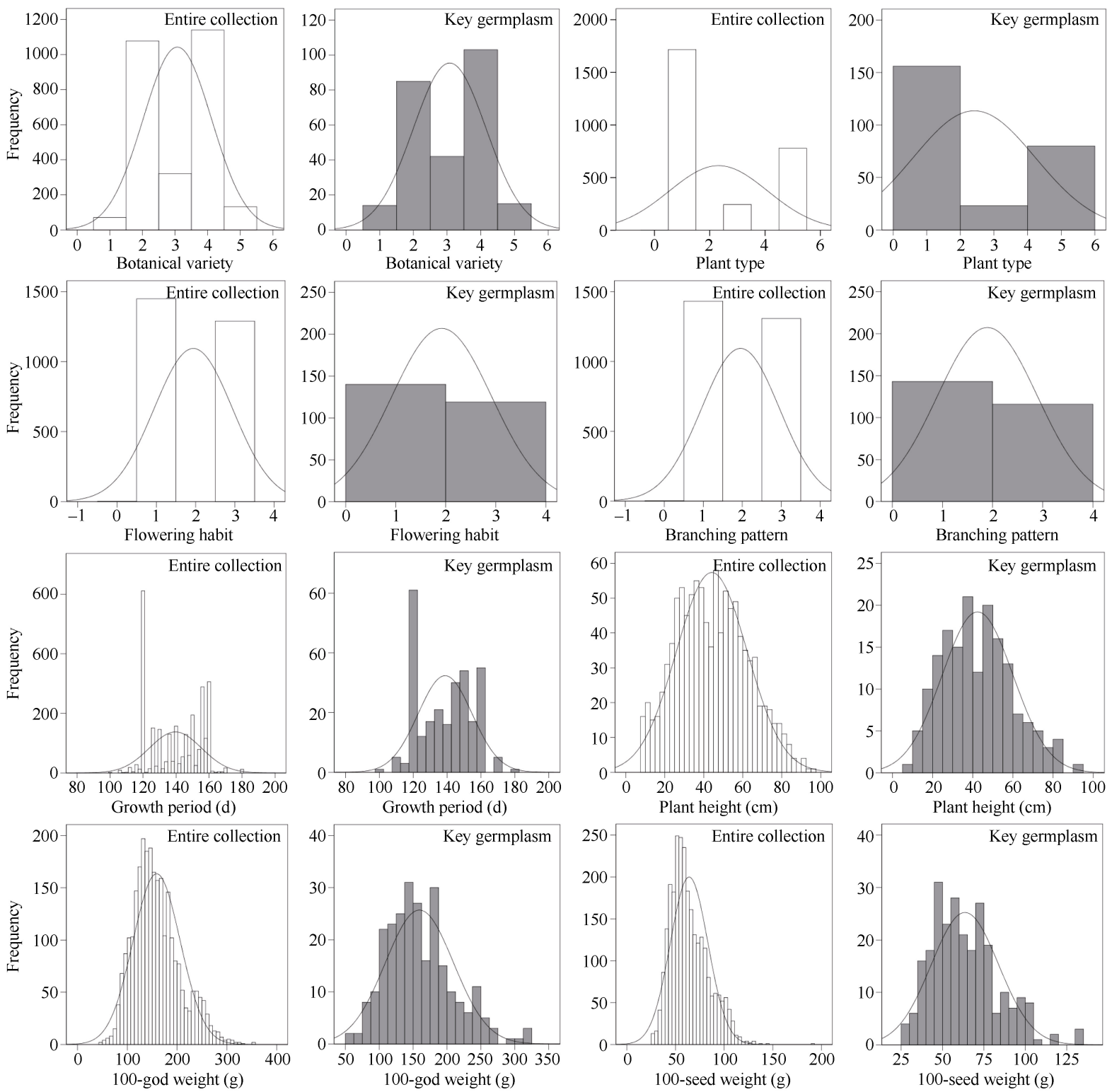

(图 2) 

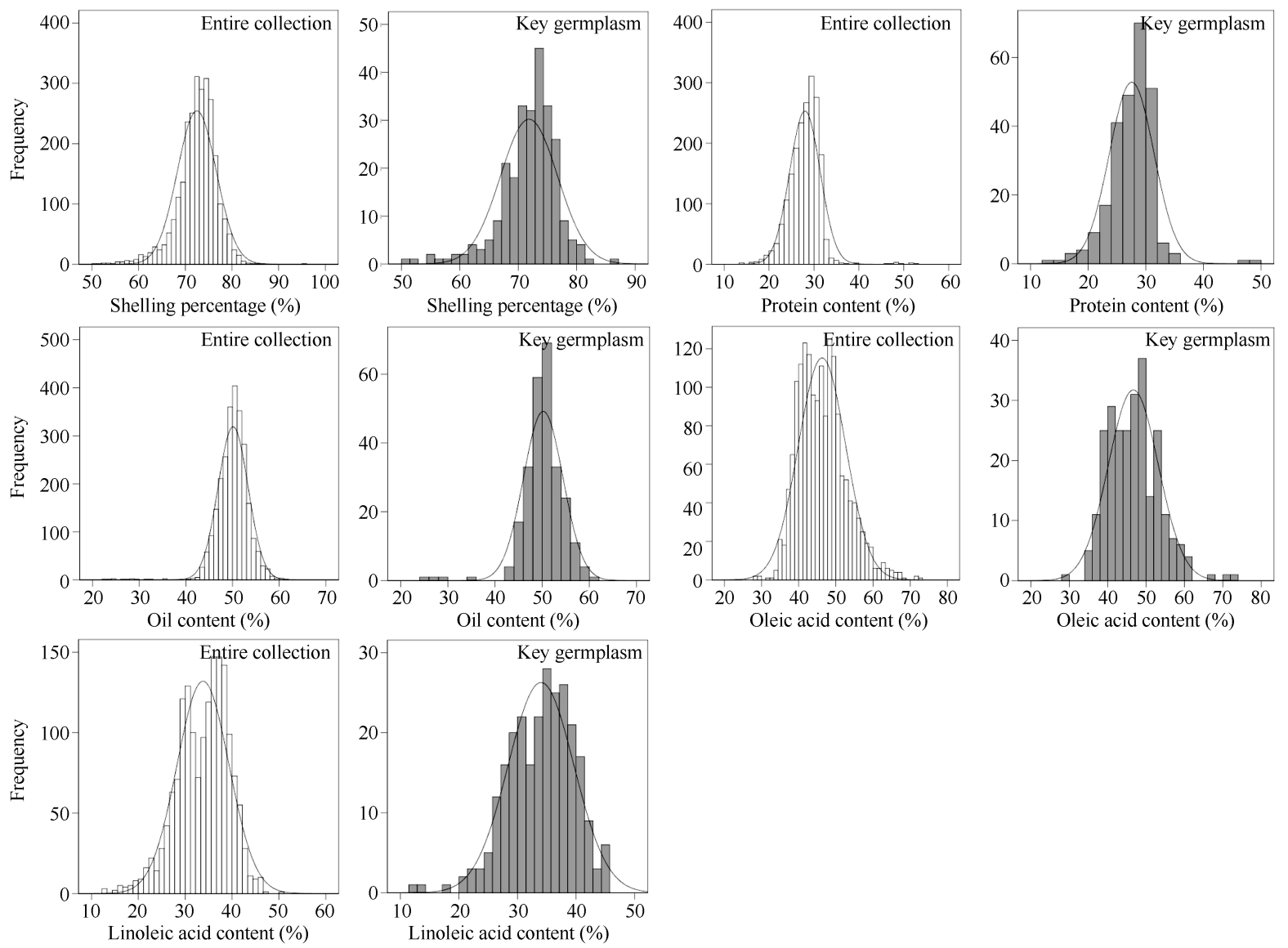

图 2 全部种质与骨干种质的 13 个相关性状频次直方图

Fig. 2 Histograms for 13 related traits of the entire collection and key germplasm

\section{3 讨论}

\section{1 取样策略对骨干种质构建的影响}

要建立质量高、代表性强的骨干种质, 采用的 取样策略很关键。为使构建的骨干种质能更有效地 代表全部种质最大的遗传多样性，一般采用 2 种取 样策略, 即逐步聚类法与分层取样+系统聚类法。前 者多见于基础数据为分子标记数据, 少见于农艺数 据。由于具有最大相似系数或最近遗传距离的种质 能够聚在一起, 从而篮除相同或相近的种质。如刘 娟等 ${ }^{[18]}$ 基于 ISSR 分子数据, 采用 UPGMA 多次聚类 抽样法+位点优先取样策略构建了包含 31 份种质的 新疆野杏核心种质。张春雨等 ${ }^{[19]}$ 基于 SSR 分子数据, 采用 UPGMA 多次聚类法+位点优先取样策略构建 了包含 25 份种质的新疆野苹果核心种质, 并利用基 因多样度、香农指数、主坐标及 SRAP 数据分析确 认了其代表性。徐益等 ${ }^{[20]}$ 则是将农艺性状聚类分析 和 SSR 分子标记聚类分析相结合, 遴选出 84 份黄麻 核心种质，可以最大限度地代表 300 份黄麻种质资
源的遗传多样性。刘遵春等 ${ }^{[21]}$ 基于 15 个数量性状数 据, 采用最短距离法逐步聚类+优先取样法构建了 包含 60 份材料的新疆野苹果核心种质。后者常见于 以农艺数据为基础数据的, 以分子数据的较少。考 虑到作物遗传多样性的发生、发展层次和分布极不 均匀，一般在建立核心种质之前，将资源归类，实 行系统分组取样, 从而更有效地达到以最小的重复 代表最大的遗传多样性 ${ }^{[22]}$ 。常见的分组方法基于植 物学分类、系谱起源、地理分布、农业生态区、生 长习性等。在系统分组的基础上, 基于表型或分子 标记数据计算的遗传距离进行聚类分析, 将材料划 分成不同的类群, 类内一般采用随机取样法或位点 优先取样法。潘英华等 ${ }^{[23]}$ 先按照地理分布将普通野 生水稻分组, 再利用 SSR 数据进行多次 UPGMA 聚 类, 构建了包含 351 份种质的广西普通野生稻核心 种质。常利芳等 ${ }^{[24]}$ 先将 144 份材料分成超甜玉米和 普甜玉米 2 组, 再根据 SSR 数据进行 UPGMA 聚类 分析+位点优先取样, 构建了包含 33 份种质的甜玉 
米核心种质。任丽平等 ${ }^{[25]}$ 先按品种特点、地理来源、 生长习性分组, 再结合 EST-STS 标记和 SSR 标记分 析，在 500 余份甘蓝型油菜种质中遴选出 87 份核心 种质。刘艳阳等 ${ }^{[26]}$ 根据地理来源分组, 组内比例法 聚类抽样, 结合 SSR 标记位点优先取样策略进行 UPGMA 逐步聚类, 在 5020 份芝麻种质资源中遴选 出 501 份核心种质。本研究按中国花生地方品种的 七大地理种植区划和五大植物学类型, 将全部种质 划分为 26 个组, 再根据 13 个性状对组内材料进行 UPGMA 聚类分析, 类内随机取样, 构建的骨干种 质的均值、方差、极差、变异系数、香农指数、分 布频率、表型保留比例等与全部种质基本无显著差 异, 且很好地保留了全部种质的表型相关性。

\section{2 组内取样量与取样比例的确定}

作物遗传结构的差异, 导致在分组聚类的基础 上组内取样量的确定方法也各不相同。常用的有平 方根法、比例法、对数法及多样性法。刘三才等 ${ }^{[27]}$ 针对普通小麦核心种质进行抽样方法比较表明, 平 方根法能提高优良类别的频率, 有利于实现小麦核 心种质在育种上的利用。此外, 合理的取样比例也 是构建骨干种质的重要环节, 其大小与原始群体的 数量规模、评价数据类型及物种遗传结构有关。目 前，国内外所构建各类作物核心种质的取样比例基 本为 $5 \% \sim 30 \%$, 一般在 $10 \%$ 左右。Brown ${ }^{[28]}$ 提出样品 数不少于 3000 时, 以 $5 \%$ 10\%的取样比例就可以代 表原始群体 $70 \%$ 的变异。Reddy 等 ${ }^{[29]}$ 构建鹰嘴豆核 心种质过程中, 原始种质为 16,991 份, 核心种质为 1956 份, 取样比例约为 $10 \%$ 。Diwan 等 ${ }^{[30]}$ 对美国一 年生苜宿资源的研究表明, 7\%是适宜的筛选比例。 魏兴华等 ${ }^{[31}$ 研究 450 份浙江籼稻地方种质的变异, 建立了 $12.5 \%$ 的核心种质。Zewdie 等 ${ }^{[32]}$ 构建的高粱 核心种质也采用了 $10 \%$ 的取样比例。本研究从 2741 份花生地方品种中选取了 242 份骨干种质, 初步评 价后补充极值材料和特殊性状种质 17 份, 取样比例 为 $9.4 \%$, 基本符合核心种质的规模。

\section{3 骨干种质的评价与确认}

骨干种质的评价就是检验其代表性和有效性。 李自超等 ${ }^{[33]}$ 认为遗传多样性指数、表型方差、表型 分布频率、变异系数、表型保留比率等是衡量骨干 种质的重要参数。本研究通过 $t$ 检验、 $F$ 测验、卡方 测验对骨干种质与全部种质比较表明, 多数性状的 均值、方差、变异系数、香农指数、分布频率等无 显著差异; 除百仁重和出仁率外, 大部分性状的变
异范围均保留在骨干种质中。另外, 二者在植物学 组成 $\left(\chi^{2}=1.600, P=0.809\right)$ 、生态分布 $\left(\chi^{2}=5.232\right.$, $P=0.514)$ 上也有很好的一致性, 表型保留比例和表 型相关性都得到了稳定的保持, 可见本研究构建的 骨干种质代表了全部种质的遗传多样性。出仁率的 方差在 2 个群体间较大, 可能与出仁率的数据缺失 较多有关。骨干种质保留了百仁重 $64.3 \%$ 的变异范 围, 可适当补充部分超大仁材料; 出仁率的保留范 围为 $77.2 \%$, 是由一份出仁率 $95.20 \%$ 的种质比同级 其他种质高出约 $10 \%$ 所致, 差异在可接受范围内。 利用主成分分析和直方图对骨干种质进一步确认, 259 份种质的遗传冗余较小，离散程度和分布特点 与全部种质一致; 骨干种质的性状变异广泛, 分布 频率与全部种质较符合, 证实了骨干种质的代表性 和有效性。

\section{4 结论}

基于种植区划和植物学类型分组, 平方根法确 定取样量, 组内按表型数据进行 UPGMA 聚类分析, 类内随机取样, 构建了中国花生地方品种的 259 份 骨干种质，占全部种质的 $9.4 \%$ ，其均值、方差、极 差、变异系数、香农指数、表型保留比例等与全部 种质无显著差异, 植物学类型组成和生态分布一致, 且保持了全部种质的表型分布频率和表型相关性。 主成分分析和直方图进一步确认了骨干种质的遗传 多样性和群体结构。本研究建立的骨干种质具有很 好的代表性。

\section{References}

[1] Kochert G, Halward T, Branch W D, Simpson C E. RFLP variability in peanut (Arachis hypogaea) cultivars and wild species. Theor Appl Genet, 1991, 81: 565-570.

[2] 姜慧芳, 任小平, 廖伯寿, 黄家权, 陈本银. 中国花生核心种 质的建立. 武汉植物学研究, 2007, 25: 289-293.

Jiang H F, Ren X P, Liao B S, Huang J Q, Chen B Y. Establishment of peanut core collection in China. $J$ Wuhan Bot Res, 2007, 25: 289-293 (in Chinese with English abstract).

[3] 沈一, 鄂志国, 刘永惠, 陈志德. 中国花生品种及其系谱数据 库的构建. 中国油料作物学报, 2015, 37: 571-575.

Shen Y, E Z G, Liu Y H, Chen Z D. Database construction of Chinese peanut varieties and their genealogy. Chin J Oil Crop Sci, 2015, 37: 571-575 (in Chinese with English abstract).

[4] 姜慧芳, 段乃雄. 花生种质资源在育种中的利用. 中国种业, 1998, (2): 24-25.

Jiang H F, Duan N X. The application of germplasm resources in peanut breeding. China Seed Ind, 1998, (2): 24-25 (in Chinese with English abstract).

[5] Frankel O H, Brown A H D. Plant genetic resources today: a 
critical appraisal. In: Holden J H W, Williams J T, eds. Crop Genetic Resources: Conservation and Evaluation. London: George Allen and Unwin, 1984. pp 249-257.

[6] 贾继增, 高丽锋, 赵光耀, 周文斌, 张卫健. 作物基因组学与 作物科学革命. 中国农业科学, 2015, 48: 3316-3332.

Jia J Z, Gao L F, Zhao G Y, Zhou W B, Zhang W J. Crop genomics and crop science revolutions. Sci Agric Sin, 2015, 48: 3316-3332 (in Chinese with English abstract).

[7] Holbrook C C, Anderson W F, Pittman R N. Selection of a core collection from the U.S. germplasm collection of peanut. Crop Sci, 1993, 33: 859-861.

[8] Holbrook C C, Dong W B. Development and evaluation of a mini core collection for the U.S. peanut germplasm collection. Crop Sci, 2005, 45: 1540-1544.

[9] Upadhyaya H D, Bramel P J, Ortiz R, Singh S. Developing a mini core of peanut for utilization of genetic resources. Crop Sci, 2002, 42: 2150-2156.

[10] Upadhyaya H D, Ortiz R, Bramel P J, Sube S. Development of a groundnut core collection using taxonomical, geographical and morphological descriptors. Genet Resour Crop Evol, 2003, 50: 139-148.

[11] Chamberlin K D C, Hassan A M, Mark E P. Evaluation of the U.S. peanut mini core collection using a molecular marker for resistance to Sclerotinia minor Jagger. Euphytica, 2010, 172: 109-115.

[12] Mukri G, Hajisaheb L, Nadaf R S B, Gowda M V C, Upadhyaya H D, Sujay V. Phenotypic and molecular dissection of ICRISAT mini core collection of peanut (Arachis hypogaea L.) for high oleic acid. Plant Breed, 2012, 131: 418-422.

[13] Upadhyaya H D, Dwivedi S L, Vadez V, Hamidou F, Singh S, Varshney R K, Liao B. Multiple resistant and nutritionally dense germplasm identified from mini core collection in peanut. Crop Sci, 2013, 54: 679-693.

[14] Sudini H, Upadhyaya H, Reddy S V, Mangala U N, Kumar K V. Resistance to late leaf spot and rust diseases in ICRISAT's mini core collection of peanut (Arachis hypogaea L.). Aust Plant Pathol, 2015, 44: 557-566.

[15] Hovav R, Badani H, Ginzberg I, Hovav R, Badani H, Ginzberg I, Chedvat I, Brand Y, Galili S. Evaluation of a peanut collection for shell-colour traits in two diverse soil types. Plant Breed, 2012, 131: 148-154.

[16] 黄莉, 任小平, 张晓杰, 陈玉宁, 姜慧芳. ICRISAT 花生微核心 种质农艺性状和黄曲霉抗性关联分析. 作物学报, 2012, 38: 935-946.

Huang L, Ren X P, Zhang X J, Chen Y N, Jiang H F. Association analysis of agronomic traits and resistance to Aspergillus flavus in the ICRISAT peanut mini-core collection. Acta Agron Sin, 2012, 38: 935-946 (in Chinese with English abstract).

[17] 任小平, 廖伯寿, 张晓杰, 雷永, 黄家权, 晏立英, 陈玉中, 姜 慧芳. 中国花生核心种质中高油酸材料的分布和遗传多样性. 植物遗传资源学报, 2011, 12: 513-518.

Ren X P, Liao B S, Zhang X J, Lei Y, Huang J Q, Yan L Y, Chen Y Z, Jiang H F. Distributing and genetic diversity of high oleic acid germplasm in peanut (Arachis hypogaea L.) core collection of China. J Plant Genet Resour, 2011, 12: 513-518 (in Chinese with English abstract).
[18] 刘娟, 廖康, 赵世荣, 曹倩, 孙琪, 刘欢. 利用 ISSR 分子标记 构建新疆野杏核心种质资源. 中国农业科学, 2015, 48: 2017-2028.

Liu J, Liao K, Zhao S R, Cao Q, Sun Q, Liu H. The core collection construction of Xinjiang wild apricot based on ISSR molecular markers. Sci Agric Sin, 2015, 48: 2017-2028 (in Chinese with English abstract).

[19] 张春雨, 陈学森, 张艳敏, 苑兆和, 刘遵春, 王延龄, 林群. 采 用分子标记构建新疆野苹果核心种质的方法. 中国农业科学, 2009, 42: 597-604.

Zhang C Y, Chen X S, Zhang Y M, Yuan Z H, Liu Z C, Wang Y L, Lin Q. A method for constructing core collection of Malussieversii using molecular markers. Sci Agric Sin, 2009, 42: 597-604 (in Chinese with English abstract).

[20] 徐益, 张列梅, 郭艳春, 祁建民, 张力岗, 方平平, 张立武. 黄 麻核心种质的遴选. 作物学报, 2019, 45: 1672-1681.

Xu Y, Zhang L M, Guo Y C, Qi J M, Zhang L L, Fang P P, Zhang $\mathrm{L}$ W. Core collection screening of a germplasm population in jute (Corchorus spp.). Acta Agron Sin, 2019, 45: 1672-1681 (in Chinese with English abstract).

[21] 刘遵春, 张春雨, 张艳敏, 张小燕, 吴传金, 王海波, 石俊, 陈 学森. 利用数量性状构建新疆野苹果核心种质的方法. 中国 农业科学, 2010, 43: 358-370.

Liu Z C, Zhang C Y, Zhang Y M, Zhang X Y, Wu C J, Wang H B, Shi J, Chen X S. Study on method of constructing core collection of Malussieversii based on quantitative traits. Sci Agric Sin, 2010, 43: 358-370 (in Chinese with English abstract).

[22] Zeuli P L S, Qualset C O. Evaluation of five strategies for obtaining a core subset from a large genetic resource collection of durum wheat. Theor Appl Genet, 1993, 87: 295-304.

[23] 潘英华, 徐志健, 梁云涛. 广西普通野生稻群体结构解析与核 心种质构建. 植物遗传资源学报, 2018, 19: 498-509.

Pan Y H, Xu Z J, Liang Y T. Genetic structure and core collection of common wild rice (Oryza rufipogon Griff.) in Guangxi. $J$ Plant Genet Resour, 2018, 19: 498-509 (in Chinese with English abstract).

[24] 常利芳, 白建荣, 李锐, 张丛卓, 张效梅, 杨瑞娟. 基于 SSR 标 记构建甜玉米群体的核心种质. 玉米科学, 2018, 26(3): 40-49.

Chang L F, Bai J R, Li R, Zhang C Z, Zhang X M, Yang R J. Construction of a core collection of sweet corn populations based on SSR markers. J Maize Sci, 2018, 26(3): 40-49 (in Chinese with English abstract).

[25] 任丽平, 倪西源, 黄吉祥, 雷伟侠, 曹明富, 赵坚义. 甘蓝型 油菜一个代表性核心种质的遴选. 中国农业科学, 2008, 41: 3521-3531.

Ren L P, Ni X Y, Huang J X, Lei W X, Cao M F, Zhao J Y. Core collection of a representative germplasm population in Brassica napus. Sci Agric Sin, 2008, 41: 3521-3531 (in Chinese with English abstract).

[26] 刘艳阳, 梅鸿献, 杜振伟, 武轫, 郑永战, 崔向华, 郑否. 基于 表型和 SSR 分子标记构建芝麻核心种质. 中国农业科学, 2017, 50: 2433-2441.

Liu Y Y, Mei H X, Du Z W, Wu K, Zheng Y Z, Cui X H, Zheng L. Construction of core collection of sesame based on phenotype and molecular markers. Sci Agric Sin, 2017, 50: 2433-2441 (in Chinese with English abstract). 
[27] 刘三才, 曹永生, 郑殿升, 刘春华, 陈梦英. 普通小麦核心种 质抽样方法的比较. 麦类作物学报, 2001, 21(2): 42-45.

Liu S C, Cao Y S, Zheng D S, Liu C H, Chen M Y. Comparing of strategies for developing core collection from common wheat. $J$ Triticeae Crops, 2001, 21(2): 42-45 (in Chinese with English abstract).

[28] Brown A H D. Core collections: a practical approach to genetic resources management. Genome, 1989, 31: 818-824.

[29] Reddy L J, Upadhyaya H D, Gowda C L L, Sube S. Development of core collection in pigeonpea [Cajanus cajan (L.) Millspaugh] using geographic and qualitative morphological descriptors. Genet Resour Crop Evol, 2005, 52: 1049-1056.

[30] Diwan N, McIntosh M S, Bauchan G R. Methods of developing a core collection of annual Medicago species. Theor Appl Genet, 1995, 90: 755-761.

[31] 魏兴华, 颜启传, 应存山, 张丽华, 章林平. 建立浙江地方
籼型稻种资源的核心样品的研究. 中国水稻科学, 1999, 13(2): 81-85.

Wei X H, Yan Q C, Ying C S, Zhang L H, Zhang L P. A core collection of Zhejiang traditional indica rice germplasm. Chin Rice Sci, 1999, 13(2): 81-85 (in Chinese with English abstract).

[32] Zewdie Y, Tong N, Bosland P. Establishing a core collection of Capsicum, using a cluster analysis with enlightened selection of accessions. Genet Resour Crop Evol, 2004, 51: 147-151.

[33] 李自超, 张洪亮, 曹永生, 穒宗恩, 魏兴华, 汤圣祥, 余萍, 王 象坤. 中国地方稻种资源初级核心种质取样策略研究. 作物 学报, 2003, 29: 20-24.

Li Z C, Zhang H L, Cao Y S, Qiu Z E, Wei X H, Tang S X, Yu $\mathrm{P}$, Wang $\mathrm{X} K$. Studies on the sampling strategy for initial core collection of Chinese ingenious rice. Acta Agron Sin, 2003, 29: 20-24 (in Chinese with English abstract). 